\title{
Development of the method of surgical correction of insolvency bauginia damper-Experience of 600 operations
}

\section{Introduction}

More than four centuries have passed since Kaspar Baugin described the existence of a valve between the small and large intestines in 1579, which was later named Bauginieva in his honor. Externally, the ileocecal flap is a cylinder that penetrates into the lumen of the cecum and has upper and lower lips, from which high folds of the membrane, called the frenulum, extend to the cecum in the anterior and posterior directions. An important component of the ileocecal barrier is the cecum itself. Its significance lies in the fact that antiperistalsis moves the contents of the intestine past the confluence of the small intestine. Thus, the maximum mechanical load falls on the cecum, which is, as it were, an "outlet" that protects the Bauhinia valve from direct mechanical impact. If the small intestine fell into the bottom of the caecosa, then the mechanical load on the ileocecal obturator would increase many times and lead to reflux in most people. The predominance of sensory neurons in the innervation of the ileocecal region indicates that the ileocecal valve is a highly sensitive formation of the human intestine. According to LG Peretz [1975], $1 \mathrm{ml}$ of the small intestine contains up to 5000 microbes, and $1 \mathrm{~g}$ of the contents of the large intestine contains about 30-40 billion. With the loss of the barrier function of the Bauhinia valve, colonization of the small intestine by foreign microbes from another habitat occurs with the development of dysbiosis ${ }^{1}$ and certain pathological consequences. ${ }^{2}$ The study of the content of enzymes in the feces of patients with reflux - enteritis showed the presence of hyperfermentosis, i.e., an increased content of enterokinase, alkaline phosphatase, as well as bile acids, pancreatic enzymes, and stomach enzymes. This fecal hyperfermentosis is a consequence of incomplete deactivation of intestinal enzymes, deconjugation of primary bile acids. Fecal hyperfermentosis, as well as the presence of primary bile acids in the large intestine, especially in its left half, serves as a trigger for spastic colitis, especially left-sided. ${ }^{3}$ M. Brotman [348] found that about $95 \%$ of bile acids are normally absorbed in the ileum, which re-enter the liver, GG Nemsadze \& EP Rybin ${ }^{4}$ found in a group of 192 patients with colon cancer a significant increase in the excretion of bile acids with the failure of the Bauhinia valve in comparison with its normal function. Indirect data on the possible role of NBZ in increasing the excretion of bile acids were obtained by analyzing the characteristics of the metabolism of bile acids in the enterohepatic cycle. ${ }^{5}$ Some authors suggest that, through the formation of carcinogenic substances from bile acids, bacteroids may contribute to an increased incidence of colon cancer. ${ }^{6}$ Other authors ${ }^{7}$ definitely believe that "the elimination of NBZ and the use of anti reflux anastomosis open up the possibility of surgical prevention of precancerous diseases and colonic cancer.

Over the past 10 years, there have been isolated publications on new techniques of bauginoplasty. The use of allografts for plastic surgery on the ileocecal junction looks promising. But it is impossible to predict how the prosthesis will behave in the long-term postoperative period. With good immediate results of the operation, the occurrence of intestinal fistulas in 14 years after the intervention and other complications have been described. ${ }^{8,9}$ Intestinal fistulas and peritonitis
Volume II Issue 6 - 2020

\author{
Martynov Vladimir Leonidovich \\ Associate professor, Department of GBUZ NO GKB No. 12 of \\ the Sormovsky, Russia
}

Correspondence: Martynov Vladimir Leonidovich, Doctor of Medical Sciences, Associate Professor, National Research Nizhny Novgorod State University named after N.I. Lobachevsky (UNN), Russia, Email hirurgia |2@mail.ru

Received: December 21, 2020 | Published: December 31, 2020

due to intestinal erosion by the prosthesis, intestinal obstruction due to the formation of adhesions between the prosthesis and the intestine have been described. ${ }^{9}$ These complications practically do not occur when using auto tissue. In addition, in the immediate postoperative period, with defects in fixation of the prosthesis, it is possible to pinch the loops of the small intestine between the prosthesis and the abdominal wall. In such cases, a greater omentum is used to delimit the prosthesis from the intestine. ${ }^{8}$ But in general, many methods of surgical correction of NBZ by various surgical techniques were unsuccessful and were abandoned.

Grekov $\mathrm{II}^{10}$ expressed a position that has retained its relevance: "Despite a number of works devoted to the proximal part of the large intestine, the pathology of this part is still insufficiently explained, because in these works the role of the Bauhinia valve was completely ignored."

\section{Surgical correction of the Bauhinia flap failure}

To eliminate NBZ, we have developed three methods of creating a locking device in the area of the ileocecal junction. The first 33 operations for NBZ were performed only by forming the lips of the valve and frenulum with serous-muscle sutures without sphincter prosthetics (RF patent No. 1790397). In 4 cases (12\%) after surgery, NBZ relapsed with resumption of reflux of the contents of the large intestine into the ileum and with the same clinical manifestations from the gastrointestinal tract that had occurred before bauginoplasty. Apparently, in these cases, reliable fusion of the sutured intestines for the formation of the valve did not occur, and in the absence of sphincters, the operations were unsuccessful. ${ }^{11,12}$

The subsequent 287 operations to create a locking device in the ileocecal region were performed with sphincter prosthetics with sections of the vascular prosthesis. No relapses were registered after these operations. The scheme of the proposed operation is shown in Figure 1.

When the cecum overflows, the large ring of the prosthesis is stretched, this leads to a slit-like change in the shape of the small 
ring, and then the ileocecal anastomosis itself, which prevents the retrograde flow of the colon contents into the ileum (RF patent No. 202664). In 18 patients, the diameter of the small ring was insufficient for the full passage of the chyme through the ileocecal junction. Of these, 9 were operated on urgently due to the development of obstruction with the imposition of ileotransverse anastomosis, which again created conditions for reflux of the contents of the colon into the small intestine. In 12 patients, transient attacks of intestinal obstruction were noted, which were stopped by conservative measures. ${ }^{13-16}$

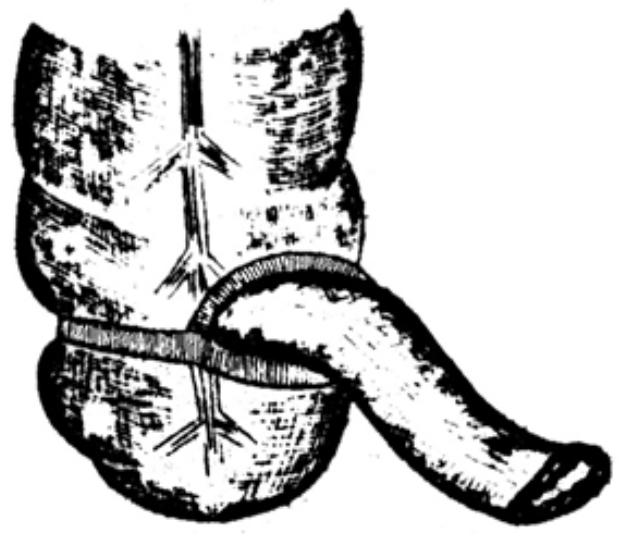

Figure I Diagram of the operation to create an ileocecal obturator with prosthetics of the sphincters Varoliy and Buzi with a strip of the vascular prosthesis.

These negative consequences of this method of bauginoplasty determined the creation of a new, simple in execution and reliable method for enhancing the are fluxity of the ileocecal junction (RF patent No. 2225173). At the beginning, the operation was performed on 10 anatomical specimens, including the terminal ileum and the right half of the colon with preliminary destruction of the Bauhinia flap. After performing the proposed operation on the preparation with water filling of the preparation, adequate filling of the cecum from the ileum and the absence of retrograde inflow of water from the cecum into the ileum were noted in all cases. The method is carried out as follows: - a laparotomy is performed with the Volkovich-Dyakonov incision or a midline laparotomy (if surgery is planned on other organs of the abdominal cavity); - the ileocecal part of the intestine is removed into the wound, a strip 5-6mm wide and up to $200 \mathrm{~mm}$ long is cut out of the vascular prosthesis (Figure 2);

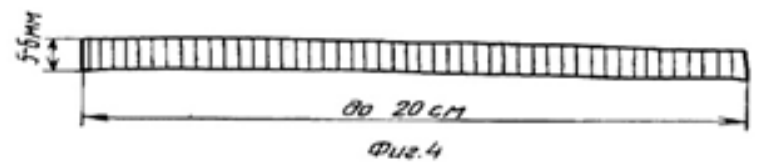

Figure $\mathbf{2}$ The strip of the vascular prosthesis required for bauginoplasty. The prosthesis is made of polyester and fluorlon threads at the Sever Production Association.

The strip of the vascular prosthesis is passed behind the ascending intestine $1-1.5 \mathrm{~cm}$ proximal to the ileocecal fistula and through the mesentery of the terminal ileum also $1-1.5 \mathrm{~cm}$ proximal to the ileocecal fistula (Figure 3);

The terminal section of the ileum for $6-7 \mathrm{~cm}$ is fixed with interrupted serous-muscular sutures for the free tape of the ascending section of the colon (Figure 4);

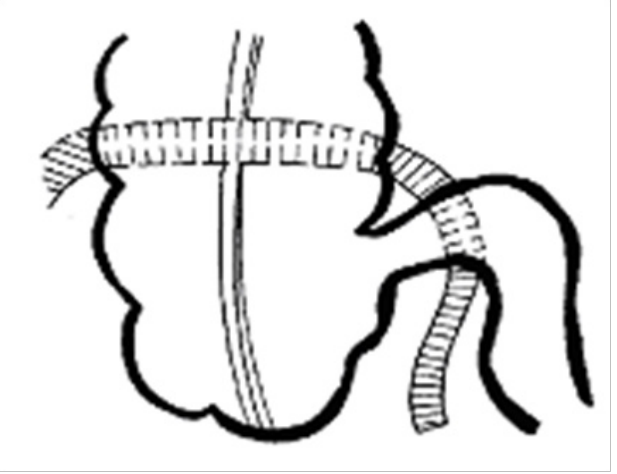

Figure 3 Stage bauginoplasty: holding the strip of the vascular prosthesis behind the ascending intestine and terminal ileum through its mesentery.

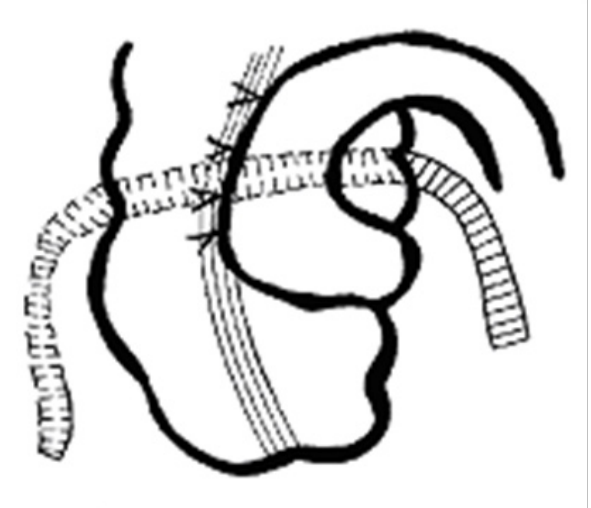

Figure 4 Stage bauginoplasty: the terminal ileum is sutured to the anterior wall of the ascending intestine.

A strip of a vascular prosthesis is placed on the anterior serous membrane of the stitched sections of the intestine $1-1.5 \mathrm{~cm}$ above the ileocecal fistula; the ends of the strip are sewn together, the excess ends of the strip are excised (Figure 5);

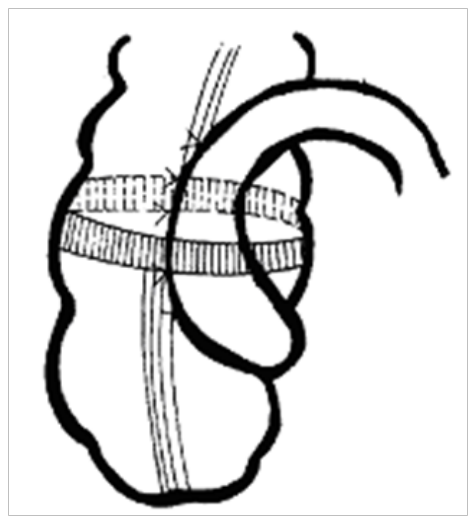

Figure 5 Stage bauginoplasty - the ends of the strip of the vascular prosthesis are stitched together, forming a ring, the diameter of which is equal to the diameter of the large intestine.

The section of the vascular prosthesis strip on the anterior surface of the ascending colon and ileum is peritonized with interrupted seromuscular sutures (Figure 6). 


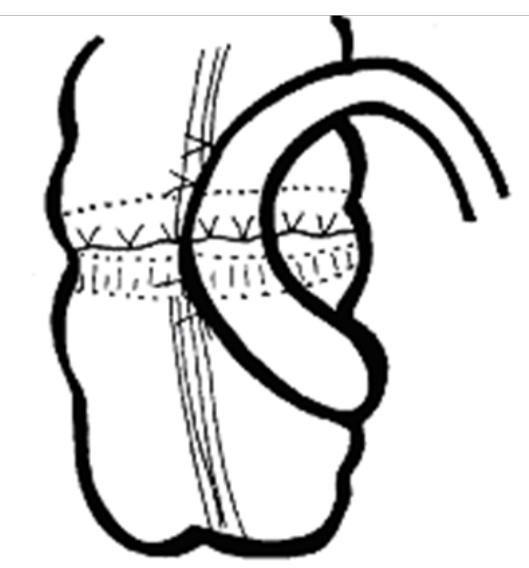

Figure 6 General view of the operation. The ring of the vascular prosthesis strip is peritoneal.

With an increase in the intracavitary pressure in the cecum and ascending colon up to and above the intracavitary pressure in the ileum, the large intestine compresses the ileum on the frame, the function of which is performed by the ring of the vascular prosthesis strip: the pressure in the lumen of the cecum and the ascending colon is not increased - the ileum is not compressed (Figure 7); the pressure in the lumen of the cecum and the ascending colon is greater than the pressure in the ileum, which is compressed by the large intestine on the frame of the vascular graft strip (Figure 8).

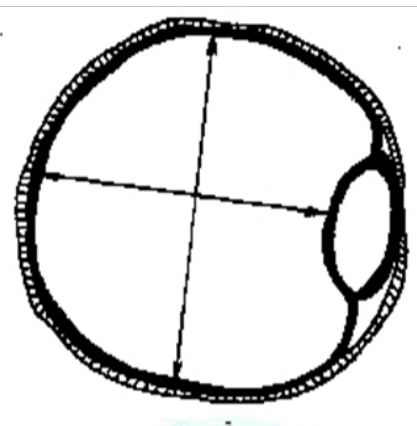

Figure 7 Diagram of an autonomously functioning structure to ensure the arefluxity of the ileocecal junction (the pressure in the lumen of the colon is lower or equal to the pressure in the lumen of the ileum, the ileum is not compressed. The contents of the small intestine can enter the colon).

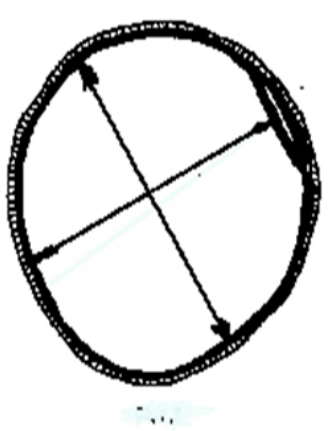

Figure 8 Scheme of an autonomously functioning structure to ensure the arefluxity of the ileocecal junction (the pressure in the lumen of the large intestine is higher than the pressure in the lumen of the small intestine, the ileum is compressed by the ascending intestine on the frame of the vascular prosthesis. The contents of the large intestine do not enter the small intestine).
Thus, a structure is created that works in an autonomous arbitrary mode, which provides the are flux function of the ileocecal obturator.

\section{3 patients with NBD were operated on using this method}

Recently, numerous reports have appeared in the literature on studies of the state of an allograft placed in the abdominal cavity on the intestinal surface.

Many authors note the phenomenon of inflammation in the intestinal wall, the formation of intestinal fistulas, migration of the prosthesis into the intestinal lumen. In two clinical observations, we also noted the migration of the strip of the vascular prosthesis into the lumen of the colon. These data made us revise the technique of bauginoplasty that we developed. Leaving the previous schematic diagram of an autonomously operating structure, the strip of the vascular prosthesis was replaced with a free isolated section of the greater omentum, through which, by injecting - puncturing, we carry out a ligature of non-absorbable polypropylene material (62 operations). Received rac. proposal No. 1385 of 08.04 .03 VMI FPS $\mathrm{RF}$. The diameter of the area of the greater omentum is $1-1.5 \mathrm{~cm}$, its length is equal to the circumference of the ascending colon (Figure 9). The ligature is designed to create a constant size of the structure, and the strand of the greater omentum to reduce the pressure of the ligature on the intestinal wall, which reduces the effect of the ligature cutting through the tissues of the intestinal wall. The final stage of the operation is shown in Figure 10. Received rac. proposal No. 1386 of 08.04.03 VMI FPS RF.

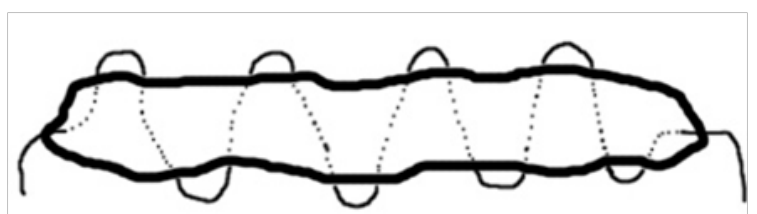

Figure 9 Diagram of a section of the greater omentum with a non-absorbable ligature passed through it.

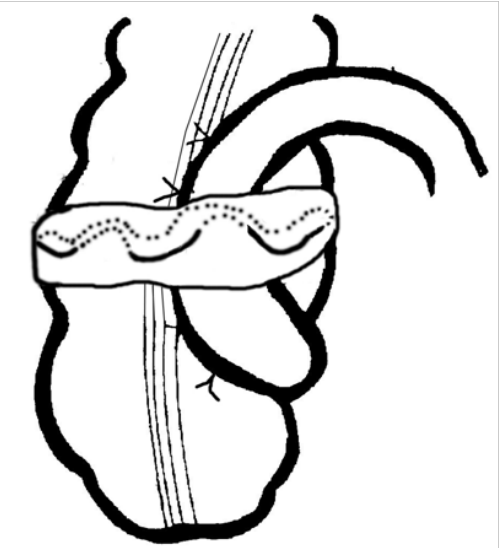

Figure 10 The final view of bauginoplasty using a large omentum section with a non-absorbable ligature passed through it.

More than 600 patients were operated on according to the technique developed by us. We hope that our approach can help find the right surgical solution.

\section{Acknowledgments}

None. 


\section{Conflicts of interest}

The author declares there are no conflicts of interest.

\section{Funding}

None.

\section{References}

1. Konev Yu V. Bowel diseases. Dysbiosis and their correction. Consilium medicum. 2005;7(6):432-437.

2. Hill M. Normal and pathological microbial flora of the upper gastrointestinal tract. Scand J Gastroenterol. 1985;111:1-6.

3. DG Mudd, ST McKelvey, W Norwood, et al. Stool bile acid concentration in patients with cancer and with an increased risk of colon cancer. Gut. 1980;21(7):587-590.

4. Nogaller AM. About food allergy. Doctor business. 1970;2:82-84.

5. Haenel H, Bendig J. Intestinal flora in health and disease. Prog Food Nutr Sci. 1975;1(1):21-64.

6. Wynder EL. The epidemiology of large cancer. Cancer Res. 1975;35:3388-3394.

7. GG Nemsadze, BA Mosidze, DT Gotsadze, et al. The functional state of the ileocecal apparatus in patients with colon cancer. Vestn Khir Im I I Grek. 1990;144(4):21-23.
8. Schumpelick V, Klinge U. Intermediate follow-up results of sublay polypropylene repair in primary and recurrent incisional hernias. Incisional hernia. 1999:313-322.

9. Wantz GE, Fisher E. Prosthetic incional hernioplasty: indications and results. Incisional hernia. 1999:303-309.

10. Grekov II. Selected works. Medicine. 1952:28-51.

11. Vitebsky Ya D. Essays on the surgery of the ileocecal section of the intestine. Medicine. 1973;111:2.

12. Goncharov PP. On visceral reflexes from the intestine. Moscow. 1941:30s.

13. Martynov. Refluxes in gastroenterology. Causes, effects, surgical treatment, prospects. Russia: Palmarium Academic Publishing; 2014:505

14. Martynov. SIBR: surgical treatment and prevention. Russia: Palmarium Academic Publishing. 2016:207.

15. Martynov V, Kolchin D, Kurilov V. Surgical prevention of reflux of the digestive system (results and scientific and practical prospects). Russia: Palmarium Academic Publishing; 2018:334.

16. Martynov VL. Prevention of the development of refluxes of the digestive system. Small intestine plug in intraoperative prevention of digestive system reflux development. Russia: Palmarium Academic Publishing; 2020:141. 